\title{
The double face of the histone variant H3.3
}

\author{
Emmanuelle Szenker ${ }^{1,2}$, Dominique Ray-Gallet ${ }^{1,2}$, Geneviève Almouzni1 ${ }^{1,2}$ \\ ${ }^{1}$ Institut Curie, Centre de Recherche, Paris F-75248, France; ${ }^{2}$ CNRS, UMR218, Paris F-75248, France
}

\begin{abstract}
Histone proteins wrap DNA to form nucleosome particles that compact eukaryotic genomes while still allowing access for cellular processes such as transcription, replication and DNA repair. Histones exist as different variants that have evolved crucial roles in specialized functions in addition to their fundamental role in packaging DNA. H3.3 - a conserved histone variant that is structurally very close to the canonical histone $\mathrm{H} 3$ - has been associated with active transcription. Furthermore, its role in histone replacement at active genes and promoters is highly conserved and has been proposed to participate in the epigenetic transmission of active chromatin states. Unexpectedly, recent data have revealed accumulation of this specific variant at silent loci in pericentric heterochromatin and telomeres, raising questions concerning the actual function of H3.3. In this review, we describe the known properties of H3.3 and the current view concerning its incorporation modes involving particular histone chaperones. Finally, we discuss the functional significance of the use of this $\mathrm{H3}$ variant, in particular during germline formation and early development in different species.
\end{abstract}

Keywords: histone variants; H3.3

Cell Research (2011) 21:421-434. doi:10.1038/cr.2011.14; published online 25 January 2011

\section{Introduction}

Histones, the main protein components of chromatin, are small basic proteins highly conserved in eukaryotes. They package and organize DNA at the level of the fundamental unit of chromatin, the nucleosome [1]. The nucleosome core particle is composed of a hetero-octamer of histones comprising a tetramer of $(\mathrm{H} 3-\mathrm{H} 4)_{2}$ flanked by two dimers of $\mathrm{H} 2 \mathrm{~A}-\mathrm{H} 2 \mathrm{~B}$, around which about $147 \mathrm{bp}$ of DNA is wrapped [2]. The dynamics of this organization permits the compaction of the genome, while enabling all cellular processes operating on DNA to occur, such as transcription, replication, recombination and repair. Nucleosomes can be modulated not only by a large variety of covalent post-translational modifications (PTMs) mostly occurring in the N-terminal tails of histones, including acetylation, phosphorylation and methylation [3] but also by the incorporation of histone variants corresponding to the histones $\mathrm{H} 3, \mathrm{H} 2 \mathrm{~A}, \mathrm{H} 2 \mathrm{~B}$ but not $\mathrm{H} 4$ for which only one form has been identified so far [4]. Histone variants were discovered on the basis of dif-

Correspondence: Geneviève Almouzni

Tel: +330156246701

E-mail: almouzni@curie.fr ferences at the level of their primary sequence that can range from a few amino acid changes to large domains. These variants show distinct regulatory mechanisms for their expression and deposition that can potentially confer specific properties to nucleosomes [5]. "Canonical histones" are defined as those with an expression peak during $\mathrm{S}$ phase to provide the main supply of histones during replication. In contrast, "replacement histones" designate those that do not show an expression peak during $\mathrm{S}$ phase. How the different histone variants are incorporated into chromatin and how they mark specific chromatin states has been a subject of intensive investigation. In this context, the study of histone chaperones, escort proteins that help to control histone supply and their incorporation into chromatin, is of interest to shed light on the specific regulation of histone variant incorporation [6].

Here, we will focus on the $\mathrm{H} 3.3$ histone $\mathrm{H} 3$ variant [7-9] to discuss the latest discoveries in this area. In mammals, five $\mathrm{H} 3$ variants have been identified: (i) two canonical variants (hereafter often referred to as H3), H3.2 and the mammalian-specific H3.1, and (ii) three replacement variants, H3.3, the centromere-specific variant CenH3 (or CENP-A in mammals) [10] and the testisspecific histone H3t [11] (Figure 1). To this list, one can add two newly characterized primate-specific $\mathrm{H} 3$ variants, H3.X and H3.Y [12]. In this review, we focus on the 


\section{A. Alignment of amino acid sequences corresponding to human $\mathrm{H} 3$ variants}

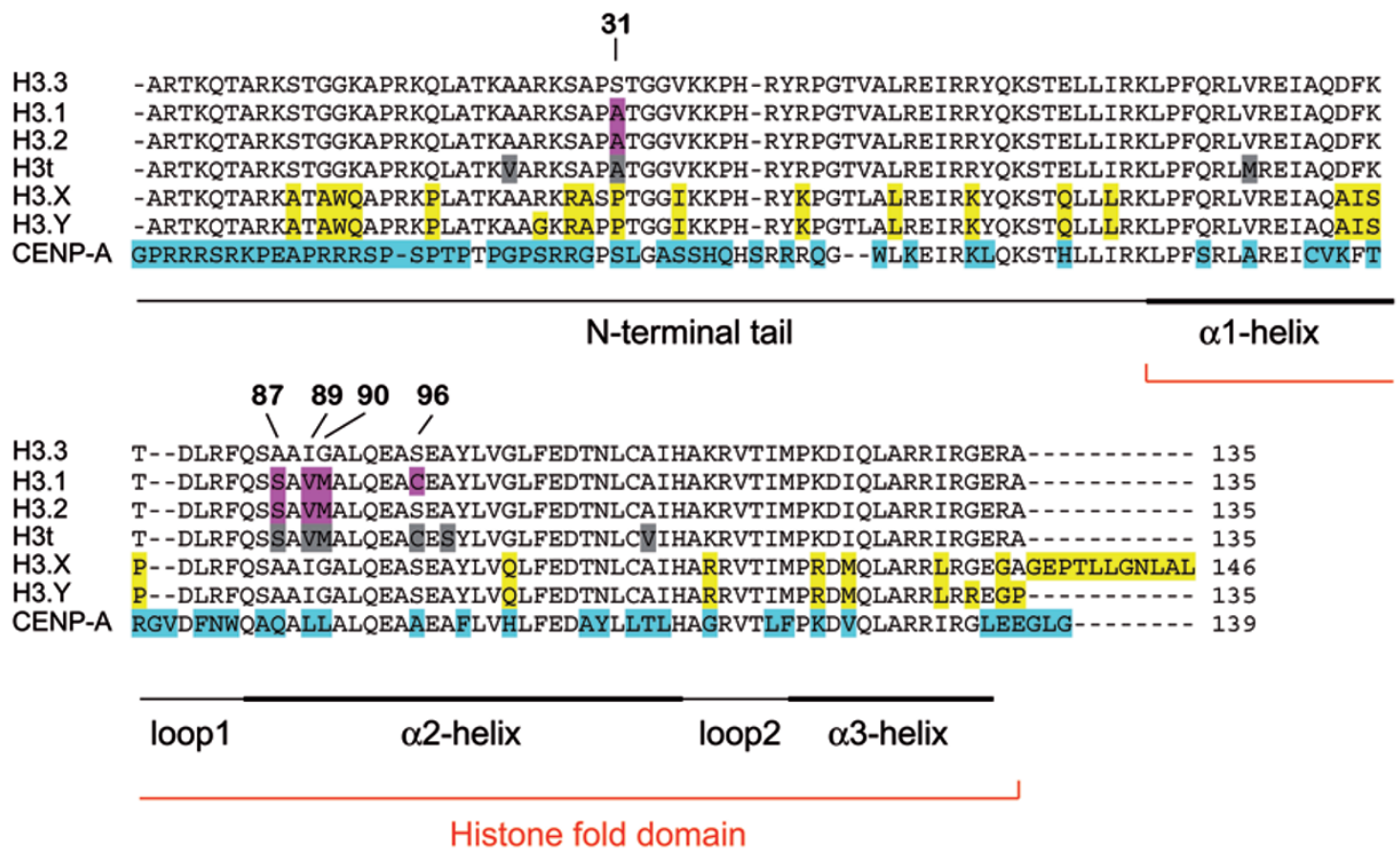

\section{B. Distinct features of human $\mathrm{H} 3$ variants}

\begin{tabular}{|c|c|c|c|c|}
\hline \multicolumn{2}{|c|}{ Histone $\mathrm{H} 3$ variants } & \multirow{2}{*}{$\begin{array}{c}\text { Expression } \\
\text { peak in S phase } \\
\text { peak in S phase }\end{array}$} & \multicolumn{2}{|c|}{$\begin{array}{l}\text { DNA-Synthesis Coupled (DSC) or DNA-synthesis } \\
\text { independent (DSI) depositions and contexts }\end{array}$} \\
\hline canonical & $\begin{array}{l}\mathrm{H} 3.1 \\
\mathrm{H} 3.2\end{array}$ & & $\begin{array}{l}\text { DSC } \\
\text { DSC }\end{array}$ & $\begin{array}{l}\text { Replication and repair } \\
\text { Replication and repair? }\end{array}$ \\
\hline replacement & $\begin{array}{c}\text { H3t } \\
\text { H3.X } \\
\text { H3.Y } \\
\text { CENP-A }\end{array}$ & $\begin{array}{c}? \\
? \\
? \\
\text { peak in } \mathrm{G} 2 \text { phase }\end{array}$ & $\begin{array}{l}- \\
- \\
- \\
\text { DSI }\end{array}$ & $\begin{array}{l}\text { Transcription } \\
\text { Male pronucleus formation } \\
\text { Meiotic Sex Chromosome Inactivation (MSCI) } \\
\text { Telomere and centromere heterochromatin } \\
\text { Tissue specific (testis) } \\
? \\
\text { Response to stress } \\
\text { Proper chromosome segregation }\end{array}$ \\
\hline
\end{tabular}

Figure 1 Sequence alignment and specific features of human H3 variants. (A) Alignment of amino acid sequences corresponding to human $\mathrm{H} 3$ variants. Sequences are compared with the "ancestral" variant $\mathrm{H} 3.3$ and the amino acid differences are highlighted. H3.1 and H3.2 differences are highlighted in purple, H3t in gray, H3.X and H3.Y in yellow, and CENP-A in light blue. The position numbers of amino acids that are different between $\mathrm{H} 3.3$ and $\mathrm{H} 3.1 / 2$ are indicated. The positions of the $\mathrm{N}$-terminal tail and of the $\alpha$-helixes of the histone-fold motif are shown. (B) Distinct features of human H3 variants. The features of canonical and replacement $\mathrm{H} 3$ variants are indicated according to their expression, mode of deposition and contexts. Canonical histones are shown in purple while H3.3 in green.

recent advances concerning the histone variant $\mathrm{H} 3.3$ to highlight its potential role in transcription and transmission of epigenetic states. Until recently, H3.3 was largely considered as a mark of transcriptional activity, for which its functional importance was under debate. While recent studies in several organisms have challenged the 
view of its specific importance during development, the unexpected enrichment of H3.3 at silent chromatin loci such as telomeres or centromeres prompts to broaden our views concerning the role of this variant. Here, we will first describe the nature, properties and regulated expression of H3.3 as compared with its canonical counterparts. Then, we will summarize the current views concerning mechanisms of H3.3 incorporation into chromatin based on recent studies. This will allow us to highlight histone chaperone complexes and major chromatin rearrangements that necessitate H3.3 deposition. Finally, we will discuss the functional relevance of the choice of a specific variant in key developmental contexts, in particular during the germline formation and early development.

\section{H3.3 properties compared with its canonical coun- terparts}

Genes encoding canonical histones such as H3.1 and H3.2 have no introns and are organized in tandem, multicopy clusters (Figure 2). Their corresponding mRNAs are not polyadenylated and their translation is tightly regulated by the binding of a particular protein, the stem loop binding protein, and of the U7 small nuclear RNA to the 3' end of the histone RNAs [13]. The peculiar genomic organization and transcriptional regulation of canonical histone genes allows a massive production at the beginning of S phase, ensuring a major supply for incorporation events during replication [14], in a DNA synthesis-coupled (DSC) manner. Of note, outside S phase, but still in a DSC manner, canonical H3.1 can also be incorporated onto chromatin at sites of DNA repair after UV lesion and possibly other damage events [15]. In contrast, genes coding for non-canonical histones are represented by single or few gene copies scattered throughout the genome (Figure 2). In addition, they often possess introns, and their mRNAs are polyadenylated. In mouse, human and Drosophila, two H3.3 genes (H3.3A and $\mathrm{H} 3.3 \mathrm{~B}$ ) encode the same conserved $\mathrm{H} 3.3$ protein, but have distinct untranslated regions [16-18]. They are expressed throughout the cell cycle, in quiescence, and are enriched in various stages of differentiation compared with their canonical counterparts [17-20]. This constitutive expression enables histone deposition/exchange through a DNA synthesis-independent (DSI) pathway during and outside $\mathrm{S}$ phase. Remarkably, this replacement variant $\mathrm{H} 3.3$ is one of the most conserved proteins present in all eukaryotes (see later, Figure 4) [21]. H3.3 has only four amino acid differences with H3.2 (at positions $31,87,89$ and 90) and five with H3.1 (with an additional difference at amino acid 96), with position 31 located in the N-terminal tail of the protein and positions
87,89 and 90 located in the $\alpha 2$ helix of the histone-fold domain (Figures 1 and 2). In spite of the high sequence similarity between $\mathrm{H} 3.3$ and $\mathrm{H} 3$, these specific residues have been proposed to account for particular properties of histone H3.3. Interestingly, serine 31 specifically found in H3.3 can be phosphorylated [22]. In vertebrates and Drosophila, residues 87, 89 and 90 are S, V and M in H3, and A, I and G in H3.3. In Drosophila, any amino acid substitution in $\mathrm{H} 3$ toward the $\mathrm{H} 3.3$ residues at these positions allows some DSI deposition of canonical H3 [23]. This result suggests that the amino acids SVM in $\mathrm{H} 3$ are critical for its restricted DSC assembly pathway. Moreover, in mouse embryonic stem (ES) cells, mutation of the endogenous H3.3B gene to the canonical H3.2 sequence alters its genome-wide enrichment patterns, supporting the importance of the amino acid sequence of H3.3 in determining its final distribution [24]. Thus, those three amino acid positions directly or indirectly provide specificity potentially via the interaction with distinct assembly machineries. Importantly, the identities of the residues found at these positions in $\mathrm{H} 3$ and $\mathrm{H} 3.3$ vary between species but always distinguish $\mathrm{H} 3$ from H3.3, arguing for a critical function of this site [21]. Furthermore, these three residues are thought to participate in the regulation of histone-histone interaction stability [25]. Indeed, H3.3 nucleosomes isolated from avian cells stably expressing tagged $\mathrm{H} 3.3$ are unusually sensitive to salt-dependent disruption, resulting in loss of their H2A/ H2B dimers [26]. Moreover, a recent study in HeLa cells expressing tagged H3.3 showed that splitting events of H3.3-H4 tetramers could be detected during DNA replication in vivo, a process that could not be observed for H3.1 [27]. The fact that H3.3 is highly enriched in actively transcribed regions raises the question whether splitting is indeed variant-specific or region-specific [28]. Taken together, these findings underscore the importance of the H3.3 sequence in addition to its cell-cycleindependent expression pattern. Additional properties of H3.3 relate to the increased proportion of PTMs associated with active chromatin such as acetylation and H3K4 methylation (Figure 2) [29-33]. How these PTMs are established on H3.3 and what roles they play in conferring an epigenetic role to this variant are crucial questions [34]. Interestingly, non-nucleosomal H3.1 and H3.3 carry a distinct set of modifications before their deposition, which in turn determine their final PTMs in nucleosomes (Figure 2) [32]. The connection between H3.3 and active transcription is further underlined by the relative depletion of the heterochromatin protein 1 (HP1) in association with H3.3-purified oligonucleosomes [32], and by the observation that $\mathrm{H} 3.3$ counteracts the association of the linker histone H1 [35]. Altogether, these data 


\section{GENOMIC ORGANISATION}

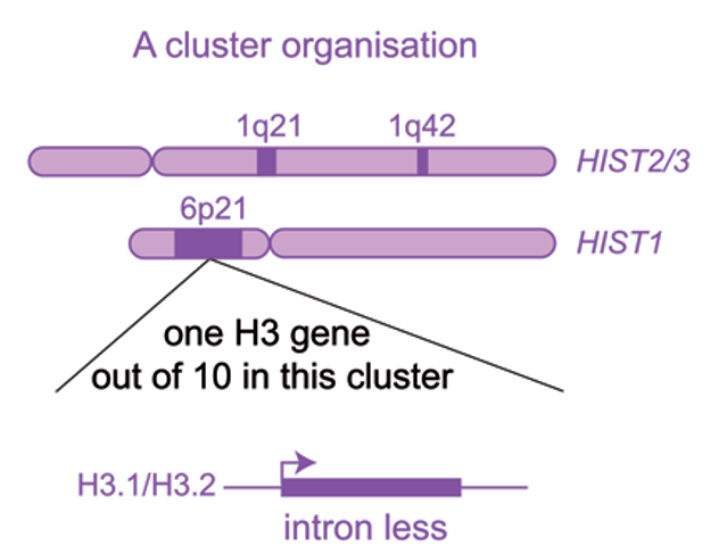

TRANSCRIPTION

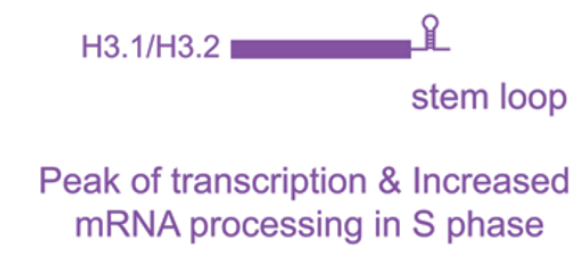

Peak of transcription \& Increased mRNA processing in $\mathrm{S}$ phase

TRANSCRI

\section{|}

|
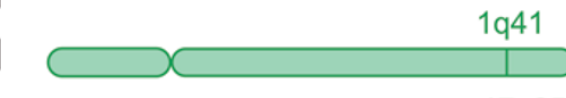

H3.3A

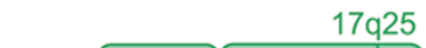

$17 q 25$

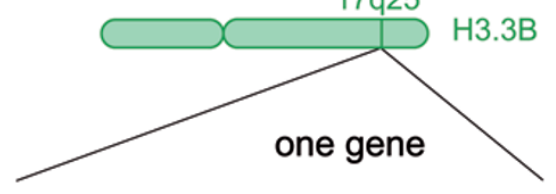

H3.3

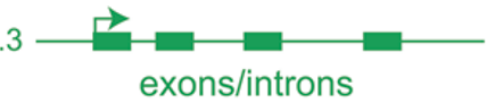

H3.3 poly A tail

Constitutive expression throughout the cell cycle

PROTEIN SEQUENCE

।

35 135 chaperone specificity?

(1)

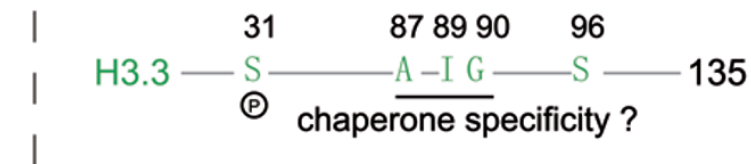

\section{ENRICHED POST-TRANSLATIONAL MARKS}

K9me1

$\mathrm{K} 9 \mathrm{me} 2 / 3$

K27me3

K64me1

\section{Before deposition}

After deposition

$\begin{array}{ll}\mid & \text { K4me2/3 } \\ \mid & \text { K36me1/2 } \\ \mid & \text { K79me1/2 } \\ \mid & \text { K122me1 } \\ \text { I } & \text { K9ac } \\ & \text { K14ac }\end{array}$

Figure 2 Human histone H3.3 compared with H3.1 and H3.2. Differences between the canonical H3 variants (H3.1 and H3.2) - in purple - and the replacement variant H3.3 - in green - are illustrated. Canonical histone genes are organized in tandem and the cluster HIST1 located on chromosome $6 \mathrm{p} 21$ contains 6 histone $\mathrm{H} 1$ and 49 core histone genes including 10 histone $\mathrm{H} 3$ genes. Canonical histone genes lack introns and are not polyadenylated in contrast to the regular genes coding for H3.3 (H3.3A and H3.3B). The amino acid differences between the canonical H3 and H3.3 are illustrated. H3.3 S31 can be phosphorylated. The motifs SVM and AIG in H3.1/2 and H3.3, respectively, could account for chaperone specificity. We also illustrate the distinct enriched marks in $\mathrm{H} 3$ and $\mathrm{H} 3.3$ before and after deposition into chromatin. 
highlight the fact that H3.3-containing nucleosomes may possess unique functional properties.

\section{H3.3 enrichment at particular genome sites}

The bulk of newly synthesized canonical histones are incorporated during DNA replication in a DSC manner, while H3.3, originally found enriched within actively transcribed genes, gets incorporated in a DSI replacement process $[23,36]$. Here, we will describe how H3.3 enrichment could be found at particular genome sites in somatic or embryonic cells and how global chromatin rearrangements in gametes and early zygotes take advantage of the use of this variant.

\section{In somatic and embryonic cells}

The use of chromatin immunoprecipitation combined with high-resolution genome mapping technologies has provided a detailed genome-wide localization profile of H3.3 in Drosophila and mammalian cells. These studies revealed specific enrichment of $\mathrm{H} 3.3$ throughout the gene body of transcribed genes as well as at the promoter regions (Figure 3) [24, 31, 36-40]. H3.3 enrichment at promoters has been observed not only at active but also at inactive genes, possibly accounting for a poised state of these genes [40, 41]. Furthermore, $\mathrm{H} 3$ replacement by the H3.3 variant also occurs at genic and intergenic regulatory regions in various metazoans [40, 42, 43]. These observations point to the possible existence of distinct roles of H3.3 linked with gene activity. A first "passive" role for H3.3 in transcription-coupled deposition could be to compensate for the eviction of nucleosomes due to the progression of the RNA polymerase complex in the body of highly transcribed genes [44]. Another "active" role could be envisaged in which H3.3 would contribute to a continuous process of histone turnover that maintains accessibility of regulatory elements to their cognate factors, which could account for the epigenetic memory of an activated state [45, 46]. Gurdon group's findings in Xenopus laevis indeed support the latter hypothesis where the presence of some "H3.3 epigenetic mark" transmitted throughout cell division rather than through a mechanism involving the reactivation of transcription at each cycle would suffice to keep the memory of an active state [46]. However, in other species such as Drosophila (embryos) or mouse (ES cells), H3.3 seems dispensable for active transcription memory. Indeed, the downregulation of H3.3 does not visibly impact upon global transcription [24, 47]. Drosophila survivors lacking H3.3 show an overexpression of $\mathrm{H} 3$ that may function in part as a compensatory mechanism. In mouse ES cells that are deficient for $\mathrm{H} 3.3$ enrichment at genic regions, the transcriptome does not exhibit dramatic changes when compared with wild-type cells [24], arguing that the presence of H3.3 is not critical for basal transcription in these cells. Whether this is stem cell-specific should be considered given that ES cells are plastic and may not necessarily stabilize a memory of activated genes in a manner comparable to differentiated cells. It would thus be of great interest to study the effect of H3.3 on the memory of an active state in differentiated mammalian cells.

In addition to its preferential accumulation at sites of active chromatin, H3.3 enrichment is also observed in particular chromosomal landmarks (Figure 3). Ahmad and Henikoff [23] found an enriched staining for H3.3GFP fusion protein that coincided with large rDNA gene repeat arrays in Drosophila Kc cells. This correlation is likely due to the presence of densely repeated genes with high transcriptional activity in the rDNA locus. Unexpectedly, enrichment of H3.3 was recently also observed in regions of the genome that should be transcriptionally silent. Indeed, H3.3 accumulation is found at telomeres and pericentric heterochromatin in mouse ES cells and mouse embryonic fibroblasts (MEF), respectively (Figure $3)[24,48-50]$. Of note, these accumulations could either reflect more loading or less removal of $\mathrm{H} 3.3$ at these loci as compared with other places in the genome. In addition, studying the proportion of $\mathrm{H} 3.3$ versus $\mathrm{H} 3$ that may influence their deposition efficiencies would need to be considered for a better understanding of the enrichment of H3.3 in different cell types, and in particular in ES cells. Accumulation of H3.3 at telomeres has so far only been described in mouse embryonic cells whereas its presence at centromeres has been previously reported in somatic cells. Indeed, in human HeLa cells, an accumulation of $\mathrm{H} 3.3$ at pericentric heterochromatin was also revealed with the use of a specific antibody recognizing H3.3 phosphorylated on serine 31 [22]. Surprisingly, in contrast to the proposed role of H3.3 in marking active chromatin, its presence at telomeres is required for the transcriptional repression of telomeric repeats [24]. While the specific functions of $\mathrm{H} 3.3$ in the organization of centromere and telomere chromatin still need to be explored, it would be of interest to illuminate whether these accumulations are also linked to transcription and whether they are essential for cell division and genome stability.

\section{In gametes and early zygotic cells}

Chromosome-wide nucleosome replacement with H3.3 incorporation occurs during mammalian meiotic sex chromosome inactivation (MSCI) in the first male meiotic prophase. This process provides a means for 


\section{H3.3 deposition pathways}

\section{Somatic and embryonic cells}

In transcribed regions and specific chromatin landmarks

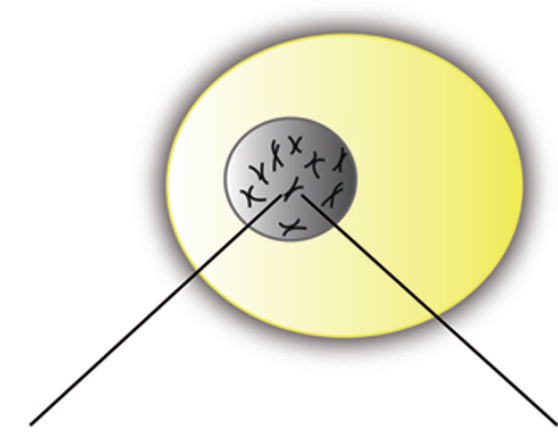

HETEROCHROMATIN
Gametes / Zygotes

In sex chromosomes during male meiosis ( $\mathrm{MSCl}$ )

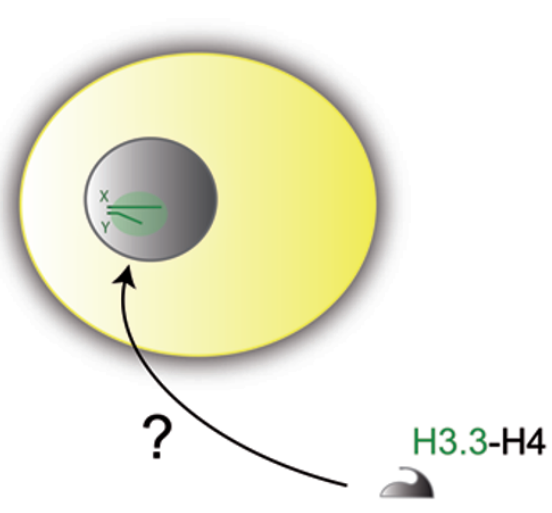

In the male pronucleus at fertilization
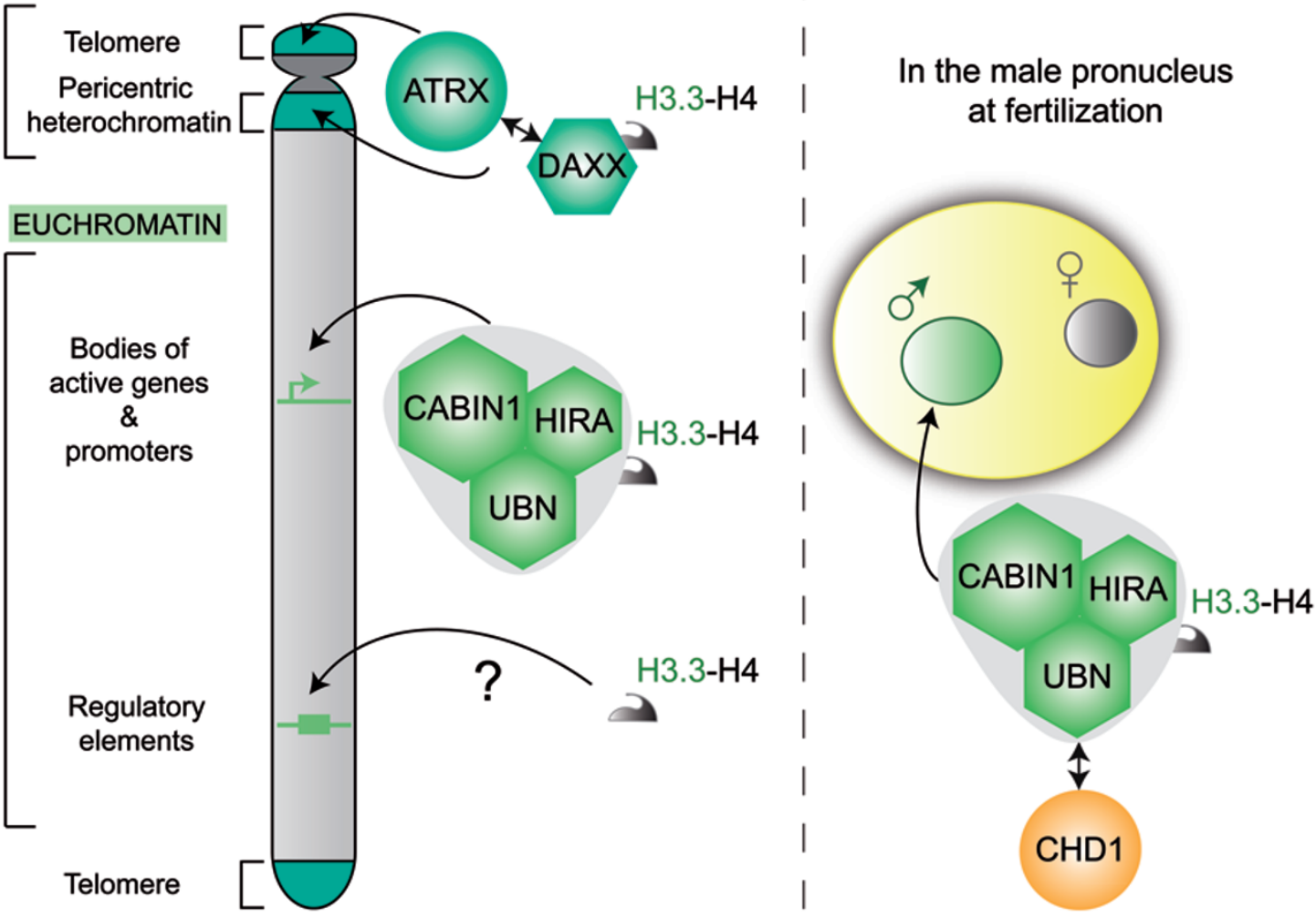

Figure 3 Local enrichment of H3.3 and complexes promoting deposition. Left: in mouse somatic and embryonic cells, H3.3 is enriched in coding regions and at specific chromatin landmarks. In heterochromatin, DAXX cooperates with the chromatin remodeler ATRX in accumulating H3.3 at pericentric heterochromatin and telomeres. It has to be noticed that accumulation of H3.3 at telomeres is so far only described in ES cells. In euchromatin, the HIRA complex mediates H3.3 enrichment in the body of transcribed genes and at promoters of transcribed or non-transcribed genes. The chaperone complex that mediates H3.3 enrichment at regulatory elements remains to be clearly identified but DAXX and/or DEK proteins have been suggested to play a role in this process. Right: in gametes, H3.3 is enriched in sex chromosomes during mouse male meiosis during MSCI. HIRA and DAXX colocalize with XY bodies but their potential role in this process still needs to be uncovered. In zygotes, H3.3 is loaded in the male pronucleus at fertilization in Drosophila and in mouse through the HIRA complex that cooperates with the chromatin remodeling factor $\mathrm{CHD} 1$. 
epigenetic reprogramming of sex chromatin presumably required for gene silencing in the male mammalian germ line [51]. In most sexually reproducing animals, another major rearrangement during spermatogenesis consists of the replacement of histones with small proteins named protamines, a process that is essential for the spermatid genome condensation into a genetically inactive state [52]. After entering the oocyte and before the formation of the diploid zygote, the sperm nucleus becomes a male pronucleus in a process that involves a series of conserved steps. Notably, a major modification of the male gamete lies in the decondensation of the highly compacted protamine-containing sperm chromatin. Concomitant with protamine removal upon fertilization, nucleosomes containing $\mathrm{H} 3.3$, but not $\mathrm{H} 3$, are specifically assembled in paternal chromatin before the first round of DNA replication in Drosophila and in mouse [53-55]. The exclusive marking of paternal chromosomes with H3.3 in the zygote represents a primary epigenetic distinction between parental genomes and underlines an important consequence of critical and highly specialized function of H3.3 loading at fertilization. In addition, H3.3 is specifically enriched in the paternal mouse pericentromeric chromatin during de novo pericentric heterochromatin formation in the male pronucleus. In this context, mutation of $\mathrm{H} 3.3 \mathrm{~K} 27$ but not $\mathrm{H} 3 \mathrm{~K} 27$ results in aberrant accumulation of pericentromeric transcripts and dysfunctional chromosome segregation [49]. This observation potentially reinforces the importance of $\mathrm{H} 3.3$ at centromeres. Interestingly, at the time of chromocenter formation in mouse early embryos, pericentric satellites undergo a transient peak in expression that is strongly biased by the parental asymmetry, an event that is necessary for proper chromocenter formation and development progression [56]. Whether the accumulation of H3.3 at centromeric regions is actually required for pericentric repeats transcription, or the converse, is worth further explorations.

H3.3 accumulation is thus observed both in active chromatin where it is proposed to participate in the epigenetic transmission of active chromatin states and in regions of the genome that should be transcriptionally silent. Whether centromeric and telomeric regions undergo transcription that is linked to H3.3 accumulation is still unclear. Knowing how H3.3 is incorporated at these specific loci and identifying the histone chaperone complexes involved in its deposition is critical to better understand the meaning of $\mathrm{H} 3.3$ enrichment patterns.

\section{H3.3 deposition and the importance of dedicated specific histone chaperones}

An important aspect of histone variant dynamics re- lates to their mode of incorporation. This mechanism involves histone chaperones and can represent a crucial step with major implications for cell fate and stability of gene expression programs. Analysis of the preassembly complexes associated with the different $\mathrm{H} 3$ variants has therefore been of interest to gain more insights in the dynamic of their incorporation and the potential histone chaperones implicated.

\section{HIRA complex}

While the study of the human canonical histone H3.1 predeposition complexes revealed the presence of the chromatin assembly factor-1 (CAF-1), the isolation of H3.3 predeposition complexes identified a distinct factor, the histone regulator A (HIRA) [57]. CAF-1, which is composed of three subunits p150, p60 and RbAp48, represents the prototype of a chaperone that promotes nucleosome assembly in a DSC pathway during replication and UV-damage repair $[58,59]$. In contrast, HIRA was described as a chaperone involved in the DSI nucleosome assembly pathway in vitro using the $X$. laevis egg extract system and shows a critical role in H3.3 deposition $[57,60]$. The genome-wide enrichment of H3.3 at promoters and in the body of active genes is affected in HIRA knockout ES cells, suggesting a critical requirement for HIRA in H3.3 deposition at these specific regions (Figure 3) [24]. Moreover, HIRA is required for H3.3 deposition in the paternal chromatin during sperm nucleus decondensation upon fertilization in Drosophila and in mouse [53, 54, 61]. HIRA might also be involved in global H3.3 incorporation in the process of MSCI as HIRA level increases in the XY body concomitantly with H3.3 deposition [51]. Accumulation of HIRA was also observed during early mouse development in primordial germ cell nuclei that undergo reprogramming to establish a distinct chromatin signature that is reminiscent of pluripotency, including the exchange of histone variants [62]. Investigating whether this HIRA behavior relates to particular rearrangement of H3.3 patterns would shed light on a general importance of H3.3 dynamics during reprogramming events. HIRA has two orthologs in Saccharomyces cerevisiae, Hir $1 \mathrm{p}$ and Hir $2 \mathrm{p}$, and biochemical purification of the Hir complex in this species revealed the presence of two co-purifying proteins Hir3 and Hpc2. Interestingly, their corresponding orthologs in human, Cabin1 and Ubinuclein (UBN1 and 2), respectively [63, 64], co-purify with human H3.3 in a sub-complex with HIRA, showing that the yeast Hir complex is conserved in human $[57,65]$. It is thus tempting to speculate that HIRA is likely to function as a complex in the process of H3.3 deposition although the respective role of each protein is still unclear. Future work should shed light on the 
mechanism at play.

\section{DAXX-ATRX complex}

Two other unexpected proteins have been specifically identified in H3.3 preassembly complexes [48]: the death domain-associated protein (DAXX), a protein originally described as being associated with FAS-mediated apoptosis [66], and the alpha-thalassemia/mental retardation X-linked syndrome protein (ATRX), a SNF2-like ATPdependent chromatin remodeling factor [67]. ATRX localizes to pericentric heterochromatin in HeLa cells [68] and was previously shown to physically associate and form a complex with DAXX in vivo [69]. DAXX was recently shown to exhibit chaperone activity and directly interact with H3.3 in a tighter manner than with H3.1 in vitro [48]. Moreover, DAXX preferentially facilitates the deposition of purified recombinant $\mathrm{H} 3.3$ onto naked DNA in "nucleosome reconstitution" assays [48, 70]. Unexpectedly, the purification of H3.3-containing complexes in DAXX ${ }^{-/-}$MEF cells identified the DSC deposition factor CAF-1 [48]. This result suggests that DAXX-deficient cells exploit H3.3 by using alternative mechanisms to bypass the loss of DAXX but further investigation is needed to understand the physiological relevance of this finding. Interestingly, ATRX co-exists in a complex with the DAXX protein, yet this complex is not required for $\mathrm{H} 3.3$ accumulation at active or repressed genes, nor at regulatory elements. Instead, this complex is specifically required for $\mathrm{H} 3.3$ enrichment at telomeres of murine ES cells $[24,70]$ and pericentric heterochromatin in MEFs [48] (Figure 3). Although only DAXX was demonstrated to display H3.3 chaperone activity [70], ATRX is also required for H3.3 accumulation at telomeres [24], suggesting that ATRX plays a role to favor H3.3 accumulation at specific chromosome landmarks. The targets of ATRX were recently shown to include Grich tandem repeats (TRs) that are found in telomeres, subtelomeric regions, rDNA, and near genes that can display altered expression patterns in the absence of ATRX [71]. However, the distribution of H3.3 at genic and intergenic TRs is only subtly perturbed when ATRX is disrupted, challenging the role of ATRX in H3.3 enrichment at these regions and its requirement for gene expression via $\mathrm{H} 3.3$ accumulation. An attractive possibility is that ATRX is required for H3.3 enrichment at a subset of TRs such as telomeres, while other proteins can intervene at genic and intergenic regions. Interestingly, DAXX can be found in complex with H3.3 in the absence of ATRX, and this complex has been proposed to mediate H3.3 deposition at regulatory elements. Additionally, DAXX was reported to accumulate in the $\mathrm{XY}$ body of mid to late stage pachytene spermatocytes, suggesting that it could be involved in the accumulation of $\mathrm{H} 3.3$ during the MSCI process, as also proposed for HIRA [72]. Lewis et al. [70] found that the interaction between DAXX and H3.3 occurs via the unique "AIG" motif at the base of the $\alpha$-helix 2 of H3.3 (Figures 1 and 2). For the first time, a peptide containing this "AIG" motif is demonstrated as necessary and sufficient for an interaction with a specific chaperone. This remarkable feature is an attractive entry point for structural studies focusing on this domain and its interaction with the other known histone $\mathrm{H} 3-\mathrm{H} 4 / \mathrm{H} 3.3-$ $\mathrm{H} 4$ chaperones, which offer promises to characterize the molecular nature of the specificity.

\section{Additional players}

Other proteins have been shown to be involved in the deposition of H3.3 at specific locations even though they are not directly found in H3.3 complexes. The remodeling factor named chromodomain helicase DNA-binding protein 1 (CHD1) physically associates with HIRA to mediate massive H3.3 incorporation into male chromatin during the decondensation of the Drosophila sperm DNA upon fertilization (Figure 3) [73]. Whether this chromatin-remodeling factor is required for other HIRA complex-mediated H3.3 depositions is an open issue. Another protein, the chromatin-bound oncogene product DEK is suggested to be an H3.3 histone chaperone in Drosophila and human cells, with potential functions to direct its deposition at regulatory elements and enhance transcription [74]. Given that DEK also associates with DAXX [75], it is possible that DEK and DAXX act together in H3.3 deposition at regulatory elements. Intriguingly, despite the importance of the HIRA complex in the deposition of H3.3 in the male pronucleus upon fertilization, HIRA is apparently dispensable for H3.3 deposition in Drosophila HIRA $^{-/}$embryos and adult cells [76]. This observation could be explained by the use of alternative mechanisms to bypass the loss of HIRA in Drosophila, potentially using distinct histone chaperones. In contrast, in mouse HIRA ${ }^{-/-}$ES cells, the accumulation of H3.3 at promoters and in the body of active genes is affected, arguing for a critical role of HIRA in H3.3 enrichment at these particular domains in mammals [24]. Altogether, these data suggest that distinct H3.3 deposition processes involving specific histone chaperone complexes have to be considered. They may act depending on the species, the developmental status and the different cell types. Moreover, the existence of alternative H3.3 deposition pathways using non-dedicated chaperones in the absence of the specific H3.3 chaperones would be an interesting compensatory mechanism that needs to be further investigated. 
Biological significances of $\mathrm{H3.3}$ deposition in various organisms

Ascomycetes, such as yeast, have only one form of non-centromeric histone $\mathrm{H} 3$, closely related to H3.3. This histone H3 (H3.3-like) present in all eukaryotes can be considered as a common ancestor. It is thus reasonable to envisage a "universal role" for this variant that can be used for both DSI and DSC nucleosome assemblies. According to the view of H3.3 as an ancestor, all noncentromeric $\mathrm{H} 3$ would derive from this original form (Figure 4). H3.2 variant present in Drosophila would be a first derivative that is already present in early branching animals such as Trichoplax. H3.1 and H3t, which are restricted to mammals, would appear later [21, 77]. H3.X and H3.Y so far only detected in primate may be restricted to this latter situation [12]. A specialization of $\mathrm{H} 3$ deposition and functions could have thus evolved while new $\mathrm{H} 3$ variants emerged. In this way, canonical H3 could specifically be restricted to DSC deposition, while H3.3 incorporation would occur mainly through a DSI pathway. Thus, an attractive hypothesis is that canonical forms of $\mathrm{H} 3$ derive from H3.3-like forms by duplication and divergence in a recurrent manner during eukaryotic evolution (Figure 4). Interestingly, so far organisms containing only a single H3-like variant have not been found. It is therefore tempting to speculate that the assembly of H3.3-containing nucleosomes may be es-

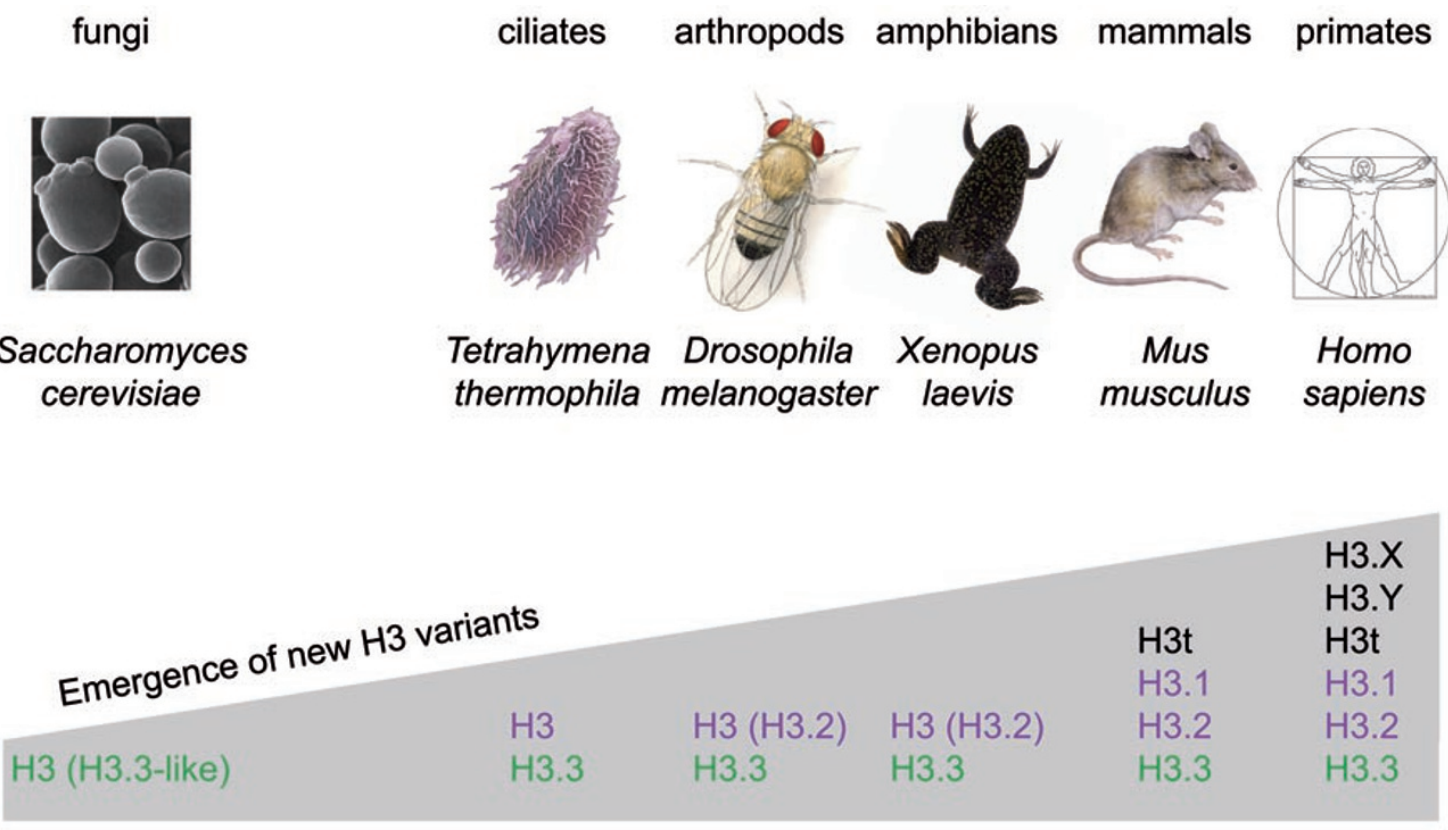

\section{Specialization of $\mathrm{H} 3$ functions}

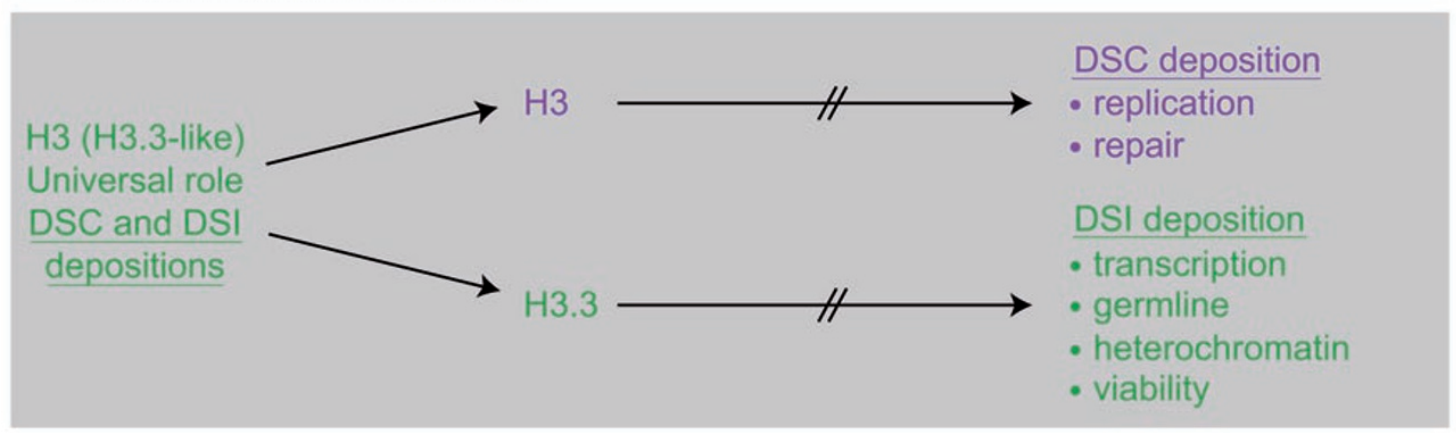

Figure 4 Emergence of non-centromeric $\mathrm{H} 3$ variants and specialization of their functions during evolution. Schematic representation of the emergence of new $\mathrm{H} 3$ histone variants concomitant with the specialization of $\mathrm{H} 3.3$ functions through evolution. Most probably, all non-centromeric H3 derive from the ancestral H3.3-like histone (green), whose functions get specialized when new H3 variants emerged, in particular the canonical H3.1 and H3.2 histones (purple). The specialization of canonical $\mathrm{H} 3$ and $\mathrm{H} 3.3$ variants functions from the universal $\mathrm{H} 3$.3-like histone is illustrated. 
sential, although additional studies may be needed to further explore this issue. In light of this hypothesis, we will present and discuss here the putative biological functions of H3.3 in different organisms from yeast to mammals.

Yeast

As mentioned above, Saccharomyces cerevisiae and Schizosaccharomyces pombe contain only one non-centromeric $\mathrm{H} 3$ variant that is related to H3.3. This H3.3-like variant is used for nucleosome assembly both outside and during $S$ phase and can therefore be deposited through both DSI and DSC pathways. In S. pombe, the deposition of H3 in a DSI manner preferentially occurs in euchromatin regions [78]. In S. cerevisiae, DSI deposition is essentially correlated with transcription [79] and this $\mathrm{H} 3$ incorporation is mainly observed at active promoters and to a lesser extent in the body of transcribed genes as well as at promoters of repressed genes [80, 81]. The pattern of yeast H3 DSI deposition thus resembles the one mediated by the HIRA complex for H3.3 incorporation in "higher" eukaryotes. Of note, in both $S$. cerevisiae and $S$. pombe, a homolog of the HIRA complex was identified with orthologs characterized for each component of this complex [82-84]. In S. cerevisiae, the Hir complex is one of the factors required for nucleosome reassembly after the passage of the RNA polymerase II, a mechanism that is proposed to play a critical role in transcription-coupled H3 deposition [85]. Moreover, given that a $S$. cerevisiae hir $\Delta$ mutant is still viable, it is possible that other chaperone complexes might replace the activity of the Hir complex or that H3 DSI deposition is not absolutely required. It would be important to try and assess whether replacing this H3.3-like histone by canonical H3 proteins can preserve viability in $S$. cerevisiae.

\section{Tetrahymena and Drosophila}

Canonical H3 is not essential in the ciliate protazoa Tetrahymena thermophila. Indeed, cells can grow without canonical H3 if H3.3 is expressed at high levels [86]. However, T. thermophila cells lacking H3.3 are viable and maintain normal nucleosome density at highly transcribed regions. Moreover, when no H3.3 is available, H3 is still not detectably deposited by the DSI pathway, indicating that DSI deposition may not be essential for survival in this organism. Strikingly though, H3.3 is required to produce viable sexual progeny and plays a critical role in the germline micronuclei late in conjugation. Along the same lines, mutations of the two H3.3 genes in Drosophila lead to partial lethality but most H3.3-deficient animals that survive to adulthood appear morphologically normal $[47,87]$. Thus, here, H3.3 is not absolutely required for viability and global development as mutant flies compensate for the lack of H3.3 in two ways: they upregulate the expression of the canonical histone $\mathrm{H} 3$ genes, and they maintain chromatin structure by using $\mathrm{H} 3$ proteins for DSI nucleosome replacement at genes. The increased expression of $\mathrm{H} 3$ is therefore sufficient to relieve transcriptional defects. However, Drosophila H3.3 is essential for fertility, as germline cells specifically require this histone variant $[47,87]$. The recent development of an in vivo genetic system that allows the replacement of deleted canonical histone genes by histone transgenes in Drosophila [88] should be most useful in order to further analyze the importance of $\mathrm{H} 3.3$ versus $\mathrm{H} 3$ in this species. Furthermore, it is interesting to realize that Drosophila paternal chromatin reorganization that necessitates the use of H3.3 at fertilization is the only developmental process that requires HIRA, a key H3.3 histone chaperone [76]. Indeed, while maternally provided HIRA is essential for global H3.3 rearrangement upon fertilization, H3.3 can be deposited in the chromatin of mutant embryos and adult cells later, suggesting that other factors are implicated in the assembly of H3.3 nucleosomes. Thus, in Tetrahymena and Drosophila, H3.3 is not required for viability and development but plays a critical role in the germline.

\section{Xenopus laevis}

Further insights into the function of H3.3 during development are provided by studies performed in the vertebrate $X$. laevis, a model organism of wide interest in developmental biology. Indeed, in contrast to Drosophila, Xenopus sperm chromatin retains $\mathrm{H} 3$ variants and H4 histones, possibly allowing sperm decondensation in the absence of global H3.3 DSI assembly. This distinct situation makes $X$. laevis an ideal model to study later roles of H3.3 during early development. The histone H3 lysine 4 methylation mark, enriched on H3.3 and correlated with transcriptional activation, has been linked to the WD40-repeat protein WDR5 as part of the methyltransferase complex associated with $\mathrm{H} 3 \mathrm{~K} 4$ trimethylation. Interestingly, WDR5-depleted $X$. laevis tadpoles exhibit a variety of developmental defects and abnormal spatial expression of Hox genes [89]. This result shows that $\mathrm{H} 3 \mathrm{~K} 4 \mathrm{me} 3$, a mark enriched on H3.3, is essential for vertebrate development. $X$. laevis is the pioneer organism in terms of animal cloning from transplanted nuclei [90]. However, the percentage of successful nuclear transfer is low and decreases when highly differentiated donor cells are used. This underlines the complexity of reprogramming a differentiated nucleus into that of an embryonic cell. After nuclear transfer, the memory of an active gene state indeed persists through numerous cell divisions in the absence of transcription and this mechanism is shown 
to depend on histone $\mathrm{H} 3.3$ incorporation into chromatin [46]. Moreover, the association with promoters of a mutated H3.3 on the methylable H3.3 lysine 4 eliminates this memory. This indicates a requirement for H3.3 K4 in the stability of gene expression patterns. Thus, incorporation of H3.3 would establish a marking system for cellular memory: after cell division, daughter cells would still maintain their patterns of gene expression by the inheritance of H3.3 at active gene loci. An emerging question now is to consider the developmental importance of this kind of memory. A key issue is thus to determine whether H3.3 is actually important for X. laevis early development.

\section{Mouse}

In mice, one of the two H3.3 genes, H3.3A, is ubiquitously expressed during embryonic development until 13.5 days post coitum and its expression is then enriched in the adult heart, kidney, brain, testes and ovaries [91]. Mutation in $\mathrm{H} 3.3 \mathrm{~A}$ results in postnatal death of $50 \%$ of homozygous mutants. Surviving animals display reduced growth rates when competing with wild-type siblings for food, exhibit neuromuscular deficits, and mutated males display reduced copulatory activity. When copulations did occur, they resulted in very few pregnancies, confirming the requirement of $\mathrm{H} 3.3$ for male fertility [91]. Thus, here, mammalian H3.3 seems not only required for reproduction but also for early development. Interestingly, HIRA is also essential for murine embryogenesis. Indeed, all homozygous HIRA $^{-/}$mutants die by day 10 or 11 of gestation. Analysis of embryos revealed an initial requirement during gastrulation, with many mutant embryos having a distorted primitive streak and patterning abnormalities of mesoendodermal derivatives prior to early embryonic lethality [92]. A deficiency in DAXX, another H3.3 chaperone, results in extensive apoptosis in early mouse development leading to embryonic lethality by day 9.5 of gestation [93]. In mice embryos null for ATRX, a partner of DAXX in dealing with H3.3, implantation and gastrulation seem normal but the embryos do not survive beyond 9.5 days post coitum due to a defect in the formation of the extraembryonic trophoblast [94]. It is important to note that these H3.3 chaperone complexes have been implicated in various cellular functions. For instance, HIRA is involved in the formation of senescence associated heterochromatin foci [95] and DAXX associates with numerous proteins involved in disparate cellular processes such as apoptosis, survival or transcriptional repression [66]. Whether the H3.3 chaperone activity is playing any role in those distinct processes and whether the observed phenotypes in null mutant mice for HIRA, DAXX and ATRX are related to their functions in
H3.3 deposition are questions that remain to be explored.

\section{Conclusions}

The replacement variant H3.3 differs from its canonical counterparts at only a few amino acid positions. Although it is still unclear whether these amino acid differences are by themselves important to modulate the chromatin organization, they are sufficient to drive H3.3 and the canonical $\mathrm{H} 3$ variants to distinct nucleosome assembly processes through the interaction with specific histone chaperones. Of note, in contrast to canonical H3, which so far uses mainly one identified chaperone, the CAF-1 complex, for incorporation into chromatin, H3.3 deposition may involve at least two different chaperone complexes, HIRA and DAXX-ATRX. The interaction with specific histone chaperones is responsible for the enrichment of the different $\mathrm{H} 3$ variants at distinct genomic sites. While the recruitment of CAF-1 at sites of DNA synthesis is likely mediated by the DNA polymerase processivity factor PCNA, ATRX is proposed to be the required component for the targeting of the DAXX-ATRX complex to telomeres or pericentric heterochromatin. How the HIRA complex is targeted to promoters and transcriptionally active genes remains to be uncovered.

Importantly, the actual role of H3.3 incorporation at specific regions of the genome is still under debate. In somatic cells, the presence of $\mathrm{H} 3.3$ was first proposed to facilitate transcription by creating a less compact chromatin. However, the importance of H3.3 enrichment for active transcription has been challenged in several organisms where normal expression of genes can occur in the absence of H3.3. Moreover, H3.3 enrichment is also observed in silent chromatin such as centromeres and telomeres, where H3.3 presence in the latter case is correlated with the repression of telomeric RNA transcription. Whether H3.3 deposition is crucial for transcriptional memory in specific contexts, in particular during development or differentiation when new expression programs have to be established, will need further exploration. In zygotes, whether global incorporation of H3.3 is an active mechanism with a specific role at fertilization or whether $\mathrm{H} 3.3$ is the only $\mathrm{H} 3$ histone available to replace the protamines and allow the decondensation of the sperm DNA is still unclear. Further investigations will be necessary to illuminate the functional relevance of $\mathrm{H} 3.3$ incorporation.

Nevertheless, specialization of H3.3 functions seems concomitant with the appearance of new $\mathrm{H} 3$ variants and the complexity of the organisms across evolution, with a specific requirement for H3.3 during development in mammals but this requirement remains to be addressed 
in Xenopus. Further work on how the H3.3-specific pathways affect chromatin structure and functions that are essential during development should help to shed light on the importance of H3.3 during evolution.

\section{Acknowledgments}

We are grateful to Armelle Corpet, Nicolas Lacoste, Zachary Gurard-Levin, Kelly Romeo and Adam Woolfe for critical reading of the manuscript and helpful discussions. The work in the authors' laboratory is supported by la Ligue Nationale contre le Cancer (Equipe labellisée Ligue 2010), PIC Programs, the European Commission Network of Excellence Epigenome (LSHGCT-2004-503433), the European Commission ITN FP7-PEOPLE-2007 "Image DDR" and FP7-PEOPLE-2008 "Nucleosome 4D", ACI-2007-Cancéropôle IdF "Breast cancer and Epigenetics", ANR "ECenS" ANR-09-BLAN-0257-01, INCa "GepiG" and ERC Advanced Grant 2009-AdG_20090506.

\section{Competing interests}

The authors declare that there is no conflict of interest.

\section{References}

1 Kornberg RD. Chromatin structure: a repeating unit of histones and DNA. Science 1974; 184:868-871.

2 Luger K, Mader AW, Richmond RK, Sargent DF, Richmond TJ. Crystal structure of the nucleosome core particle at $2.8 \mathrm{~A}$ resolution. Nature 1997; 389:251-260.

3 Kouzarides T. Chromatin modifications and their function. Cell 2007; 128:693-705.

4 Zweidler A. Core histone variants of the mouse: primary structure and differential expression. In: JL Stein GS Stein, WF Marzluff, eds. Vol. Chapter 14, Histones Genes, Structure, Organization and Regulation. John Wiley \& sons, 1984:339-371.

5 Talbert PB, Henikoff S. Histone variants - ancient wrap artists of the epigenome. Nat Rev Mol Cell Biol 2010; 11:264-275.

6 De Koning L, Corpet A, Haber JE, Almouzni G. Histone chaperones: an escort network regulating histone traffic. Nat Struct Mol Biol 2007; 14:997-1007.

7 Orsi GA, Couble P, Loppin B. Epigenetic and replacement roles of histone variant $\mathrm{H} 3.3$ in reproduction and development. Int J Dev Biol 2009; 53:231-243.

8 Elsaesser SJ, Goldberg AD, Allis CD. New functions for an old variant: no substitute for histone H3.3. Curr Opin Genet Dev 2010; 20:110-117.

9 Banaszynski LA, Allis CD, Lewis PW. Histone variants in metazoan development. Dev Cell 2010; 19:662-674.

10 Allshire RC, Karpen GH. Epigenetic regulation of centromeric chromatin: old dogs, new tricks? Nat Rev Genet 2008; 9:923937.

11 Witt O, Albig W, Doenecke D. Testis-specific expression of a novel human H3 histone gene. Exp Cell Res 1996; 229:301306

12 Wiedemann SM, Mildner SN, Bonisch C, et al. Identification and characterization of two novel primate-specific histone $\mathrm{H} 3$ variants, H3.X and H3.Y. J Cell Biol 2010; 190:777-791.

13 Marzluff WF, Gongidi P, Woods KR, Jin J, Maltais LJ. The human and mouse replication-dependent histone genes. Genomics 2002; 80:487-498.

14 Osley MA. The regulation of histone synthesis in the cell cycle. Annu Rev Biochem 1991; 60:827-861.

15 Polo SE, Roche D, Almouzni G. New histone incorporation marks sites of UV repair in human cells. Cell 2006; 127:481493.

16 Akhmanova AS, Bindels PC, Xu J, et al. Structure and expression of histone H3.3 genes in Drosophila melanogaster and Drosophila hydei. Genome 1995; 38:586-600.

17 Frank D, Doenecke D, Albig W. Differential expression of human replacement and cell cycle dependent $\mathrm{H} 3$ histone genes. Gene 2003; 312:135-143.

18 Krimer DB, Cheng G, Skoultchi AI. Induction of H3.3 replacement histone mRNAs during the precommitment period of murine erythroleukemia cell differentiation. Nucleic Acids Res 1993; 21:2873-2879.

19 Castiglia D, Cestelli A, Scaturro M, Nastasi T, Di Liegro I. H1(0) and H3.3B mRNA levels in developing rat brain. Neurochem Res 1994; 19:1531-1537.

$20 \mathrm{Wu}$ RS, Tsai S, Bonner WM. Patterns of histone variant synthesis can distinguish G0 from G1 cells. Cell 1982; 31:367374.

21 Malik HS, Henikoff S. Phylogenomics of the nucleosome. Nat Struct Biol 2003; 10:882-891.

22 Hake SB, Garcia BA, Kauer M, et al. Serine 31 phosphorylation of histone variant H3.3 is specific to regions bordering centromeres in metaphase chromosomes. Proc Natl Acad Sci USA 2005; 102:6344-6349.

23 Ahmad K, Henikoff S. The histone variant H3.3 marks active chromatin by replication-independent nucleosome assembly. Mol Cell 2002; 9:1191-1200.

24 Goldberg AD, Banaszynski LA, Noh KM, et al. Distinct factors control histone variant H3.3 localization at specific genomic regions. Cell 2010; 140:678-691.

25 Hake SB, Allis CD. Histone H3 variants and their potential role in indexing mammalian genomes: the "H3 barcode hypothesis". Proc Natl Acad Sci USA 2006; 103:6428-6435.

26 Jin C, Felsenfeld G. Nucleosome stability mediated by histone variants H3.3 and H2A.Z. Genes Dev 2007; 21:1519-1529.

27 Xu M, Long C, Chen X, et al. Partitioning of histone H3-H4 tetramers during DNA replication-dependent chromatin assembly. Science 2010; 328:94-98.

28 Ray-Gallet D, Almouzni G. Molecular biology. Mixing or not mixing. Science 2010; 328:56-57.

29 Waterborg JH. Sequence analysis of acetylation and methylation in two histone H3 variants of alfalfa. J Biol Chem 1990; 265:17157-17161.

30 McKittrick E, Gafken PR, Ahmad K, Henikoff S. Histone H3.3 is enriched in covalent modifications associated with active chromatin. Proc Natl Acad Sci USA 2004; 101:1525-1530.

31 Chow CM, Georgiou A, Szutorisz H, et al. Variant histone H3.3 marks promoters of transcriptionally active genes during mammalian cell division. EMBO Rep 2005; 6:354-360.

32 Loyola A, Bonaldi T, Roche D, Imhof A, Almouzni G. PTMs on $\mathrm{H} 3$ variants before chromatin assembly potentiate their final epigenetic state. Mol Cell 2006; 24:309-316. 
33 Hake SB, Garcia BA, Duncan EM, et al. Expression patterns and post-translational modifications associated with mammalian histone $\mathrm{H} 3$ variants. J Biol Chem 2006; 281:559-568.

34 Loyola A, Almouzni G. Marking histone H3 variants: how, when and why? Trends Biochem Sci 2007; 32:425-433.

35 Braunschweig U, Hogan GJ, Pagie L, van Steensel B. Histone $\mathrm{H} 1 \mathrm{binding}$ is inhibited by histone variant H3.3. EMBO J 2009; 28:3635-3645.

36 Schwartz BE, Ahmad K. Transcriptional activation triggers deposition and removal of the histone variant H3.3. Genes Dev 2005; 19:804-814.

37 Delbarre E, Jacobsen BM, Reiner AH, et al. Chromatin environment of histone variant $\mathrm{H} 3.3$ revealed by quantitative imaging and genome-scale chromatin and DNA immunoprecipitation. Mol Biol Cell 2010; 21:1872-1884.

38 Wirbelauer C, Bell O, Schubeler D. Variant histone H3.3 is deposited at sites of nucleosomal displacement throughout transcribed genes while active histone modifications show a promoter-proximal bias. Genes Dev 2005; 19:1761-1766.

39 Daury L, Chailleux C, Bonvallet J, Trouche D. Histone H3.3 deposition at E2F-regulated genes is linked to transcription. EMBO Rep 2006; 7:66-71.

40 Mito Y, Henikoff JG, Henikoff S. Genome-scale profiling of histone H3.3 replacement patterns. Nat Genet 2005; 37:10901097.

41 Tamura T, Smith M, Kanno T, et al. Inducible deposition of the histone variant H3.3 in interferon-stimulated genes. J Biol Chem 2009; 284:12217-12225.

42 Jin C, Zang C, Wei G, et al. H3.3/H2A.Z double variantcontaining nucleosomes mark 'nucleosome-free regions' of active promoters and other regulatory regions. Nat Genet 2009; 41:941-945.

43 Nakayama T, Nishioka K, Dong YX, Shimojima T, Hirose S. Drosophila GAGA factor directs histone H3.3 replacement that prevents the heterochromatin spreading. Genes Dev 2007; 21:552-561.

44 Schwartz BE, Ahmad K. 2. Chromatin assembly with H3 histones: full throttle down multiple pathways. Curr Top Dev Biol 2006; 74:31-55.

45 Henikoff S. Nucleosome destabilization in the epigenetic regulation of gene expression. Nat Rev Genet 2008; 9:15-26.

$46 \mathrm{Ng} \mathrm{RK}$, Gurdon JB. Epigenetic memory of an active gene state depends on histone H3.3 incorporation into chromatin in the absence of transcription. Nat Cell Biol 2008; 10:102-109.

47 Sakai A, Schwartz BE, Goldstein S, Ahmad K. Transcriptional and developmental functions of the H3.3 histone variant in Drosophila. Curr Biol 2009; 19:1816-1820.

48 Drané P, Ouararhni K, Depaux A, Shuaib M, Hamiche A. The death-associated protein DAXX is a novel histone chaperone involved in the replication-independent deposition of H3.3. Genes Dev 2010; 24:1253-1265.

49 Santenard A, Ziegler-Birling C, Koch M, et al. Heterochromatin formation in the mouse embryo requires critical residues of the histone variant H3.3. Nat Cell Biol 2010; 12:853-862.

50 Wong LH, Ren H, Williams E, et al. Histone H3.3 incorporation provides a unique and functionally essential telomeric chromatin in embryonic stem cells. Genome Res 2009; 19:404414.

51 van der Heijden GW, Derijck AA, Posfai E, et al. Chromo- some-wide nucleosome replacement and H3.3 incorporation during mammalian meiotic sex chromosome inactivation. Nat Genet 2007; 39:251-258.

52 Balhorn R. The protamine family of sperm nuclear proteins. Genome Biol 2007; 8:227.

53 Loppin B, Bonnefoy E, Anselme C, et al. The histone H3.3 chaperone HIRA is essential for chromatin assembly in the male pronucleus. Nature 2005; 437:1386-1390.

54 van der Heijden GW, Dieker JW, Derijck AA, et al. Asymmetry in histone $\mathrm{H} 3$ variants and lysine methylation between paternal and maternal chromatin of the early mouse zygote. Mech Dev 2005; 122:1008-1022.

55 Torres-Padilla ME, Bannister AJ, Hurd PJ, Kouzarides T, Zernicka-Goetz M. Dynamic distribution of the replacement histone variant H3.3 in the mouse oocyte and preimplantation embryos. Int J Dev Biol 2006; 50:455-461.

56 Probst AV, Okamoto I, Casanova M, et al. A strand-specific burst in transcription of pericentric satellites is required for chromocenter formation and early mouse development. Dev Cell 2010; 19:625-638.

57 Tagami H, Ray-Gallet D, Almouzni G, Nakatani Y. Histone H3.1 and H3.3 complexes mediate nucleosome assembly pathways dependent or independent of DNA synthesis. Cell 2004; 116:51-61.

58 Stillman B. Chromatin assembly during SV40 DNA replication in vitro. Cell 1986; 45:555-565.

59 Gaillard PH, Martini EM, Kaufman PD, et al. Chromatin assembly coupled to DNA repair: a new role for chromatin assembly factor I. Cell 1996; 86:887-896.

60 Ray-Gallet D, Quivy JP, Scamps C, et al. HIRA is critical for a nucleosome assembly pathway independent of DNA synthesis. Mol Cell 2002; 9:1091-1100.

61 Loppin B, Docquier M, Bonneton F, Couble P. The maternal effect mutation sesame affects the formation of the male pronucleus in Drosophila melanogaster. Dev Biol 2000; 222:392404.

62 Hajkova P, Ancelin K, Waldmann T, et al. Chromatin dynamics during epigenetic reprogramming in the mouse germ line. Nature 2008; 452:877-881.

63 Banumathy G, Somaiah N, Zhang R, et al. Human UBN1 is an ortholog of yeast $\mathrm{Hpc} 2 \mathrm{p}$ and has an essential role in the HIRA/ ASF1a chromatin-remodeling pathway in senescent cells. Mol Cell Biol 2009; 29:758-770.

64 Balaji S, Iyer LM, Aravind L. HPC2 and ubinuclein define a novel family of histone chaperones conserved throughout eukaryotes. Mol Biosyst 2009; 5:269-275.

65 Elsaesser SJ, Allis CD. HIRA and Daxx constitute two independent histone H3.3-containing predisposition complexes. Cold Spring Harb Symp Quant Biol 2010 Dec 7. doi:10.1101/ sqb.2010.75.008

66 Salomoni P, Khelifi AF. Daxx: death or survival protein? Trends Cell Biol 2006; 16:97-104.

67 Gibbons RJ, Picketts DJ, Villard L, Higgs DR. Mutations in a putative global transcriptional regulator cause X-linked mental retardation with alpha-thalassemia (ATR-X syndrome). Cell 1995; 80:837-845.

68 McDowell TL, Gibbons RJ, Sutherland H, et al. Localization of a putative transcriptional regulator (ATRX) at pericentromeric heterochromatin and the short arms of acrocentric chro- 
mosomes. Proc Natl Acad Sci USA 1999; 96:13983-13988.

69 Xue Y, Gibbons R, Yan Z, et al. The ATRX syndrome protein forms a chromatin-remodeling complex with Daxx and localizes in promyelocytic leukemia nuclear bodies. Proc Natl Acad Sci USA 2003; 100:10635-10640.

70 Lewis PW, Elsaesser SJ, Noh KM, Stadler SC, Allis CD. Daxx is an H3.3-specific histone chaperone and cooperates with ATRX in replication-independent chromatin assembly at telomeres. Proc Natl Acad Sci USA 2010; 107:14075-14080.

71 Law MJ, Lower KM, Voon HP, et al. ATR-X syndrome protein targets tandem repeats and influences allele-specific expression in a size-dependent manner. Cell 2010; 143:367-378.

72 Rogers RS, Inselman A, Handel MA, Matunis MJ. SUMO modified proteins localize to the XY body of pachytene spermatocytes. Chromosoma 2004; 113:233-243.

73 Konev AY, Tribus M, Park SY, et al. CHD1 motor protein is required for deposition of histone variant H3.3 into chromatin in vivo. Science 2007; 317:1087-1090.

74 Sawatsubashi S, Murata T, Lim J, et al. A histone chaperone, DEK, transcriptionally coactivates a nuclear receptor. Genes Dev 2010; 24:159-170.

75 Hollenbach AD, McPherson CJ, Mientjes EJ, Iyengar R, Grosveld G. Daxx and histone deacetylase II associate with chromatin through an interaction with core histones and the chromatin-associated protein Dek. J Cell Sci 2002; 115:33193330.

76 Bonnefoy E, Orsi GA, Couble P, Loppin B. The essential role of Drosophila HIRA for de novo assembly of paternal chromatin at fertilization. PLoS Genet 2007; 3:1991-2006.

77 Postberg J, Forcob S, Chang WJ, Lipps HJ. The evolutionary history of histone $\mathrm{H} 3$ suggests a deep eukaryotic root of chromatin modifying mechanisms. BMC Evol Biol 2010; 10:259.

78 Choi ES, Shin JA, Kim HS, Jang YK. Dynamic regulation of replication independent deposition of histone $\mathrm{H} 3$ in fission yeast. Nucleic Acids Res 2005; 33:7102-7110.

79 Jamai A, Imoberdorf RM, Strubin M. Continuous histone H2B and transcription-dependent histone $\mathrm{H} 3$ exchange in yeast cells outside of replication. Mol Cell 2007; 25:345-355.

80 Rufiange A, Jacques PE, Bhat W, Robert F, Nourani A. Genome-wide replication-independent histone $\mathrm{H} 3$ exchange occurs predominantly at promoters and implicates H3 K56 acetylation and Asf1. Mol Cell 2007; 27:393-405.

81 Dion MF, Kaplan T, Kim M, et al. Dynamics of replicationindependent histone turnover in budding yeast. Science 2007; 315:1405-1408.

82 Prochasson P, Florens L, Swanson SK, Washburn MP, Workman JL. The HIR corepressor complex binds to nucleosomes generating a distinct protein/DNA complex resistant to remodeling by SWI/SNF. Genes Dev 2005; 19:2534-2539.

83 Green EM, Antczak AJ, Bailey AO, et al. Replication-independent histone deposition by the HIR complex and Asf1. Curr Biol 2005; 15:2044-2049.

84 Anderson HE, Kagansky A, Wardle J, et al. Silencing mediated by the Schizosaccharomyces pombe HIRA complex is dependent upon the Hpc2-like protein, Hip4. PLoS One 2010; 5:e13488.

85 Formosa T, Ruone S, Adams MD, et al. Defects in SPT16 or POB3 (yFACT) in Saccharomyces cerevisiae cause dependence on the Hir/Hpc pathway: polymerase passage may degrade chromatin structure. Genetics 2002; 162:1557-1571.

86 Cui B, Liu Y, Gorovsky MA. Deposition and function of histone H3 variants in Tetrahymena thermophila. Mol Cell Biol 2006; 26:7719-7730.

87 Hodl M, Basler K. Transcription in the absence of histone H3.3. Curr Biol 2009; 19:1221-1226.

88 Gunesdogan U, Jackle H, Herzig A. A genetic system to assess in vivo the functions of histones and histone modifications in higher eukaryotes. EMBO Rep 2010; 11:772-776.

89 Wysocka J, Swigut T, Milne TA, et al. WDR5 associates with histone $\mathrm{H} 3$ methylated at $\mathrm{K} 4$ and is essential for $\mathrm{H} 3 \mathrm{~K} 4$ methylation and vertebrate development. Cell 2005; 121:859-872.

90 Gurdon JB. The developmental capacity of nuclei taken from intestinal epithelium cells of feeding tadpoles. J Embryol Exp Morphol 1962; 10:622-640.

91 Couldrey C, Carlton MB, Nolan PM, Colledge WH, Evans MJ. A retroviral gene trap insertion into the histone 3.3A gene causes partial neonatal lethality, stunded growth, neuromuscular deficits and male sub-fertility in transgenic mice. Hum Mol Genet 1999; 8:2489-2495.

92 Roberts C, Sutherland HF, Farmer H, et al. Targeted mutagenesis of the Hira gene results in gastrulation defects and patterning abnormalities of mesoendodermal derivatives prior to early embryonic lethality. Mol Cell Biol 2002; 22:2318-2328.

93 Michaelson JS, Bader D, Kuo F, Kozak C, Leder P. Loss of Daxx, a promiscuously interacting protein, results in extensive apoptosis in early mouse development. Genes Dev 1999; 13:1918-1923.

94 Garrick D, Sharpe JA, Arkell R, et al. Loss of Atrx affects trophoblast development and the pattern of X-inactivation in extraembryonic tissues. PLoS Genet 2006; 2:e58.

95 Zhang R, Chen W, Adams PD. Molecular dissection of formation of senescence-associated heterochromatin foci. Mol Cell Biol 2007; 27:2343-2358. 